\title{
An Unsuspected Ameloblastoma in the Subpontic Region of the Mandible with Consideration of Pathogenesis From the RADIOGRAPHIC COURSE
}

\author{
C. H. Siar ${ }^{1}$, K. Nakano 2 , P. I. Chelvanayagam ${ }^{3}$, K. H. Ng${ }^{4}$, H. Nagatsuka ${ }^{5}$, T. Kawakami ${ }^{2}$ \\ ${ }^{1}$ Department of Oral Pathology, Oral Medicine and Periodontology, Faculty of Dentistry, University of Malaya, Kuala Lumpur, Malaysia, \\ ${ }^{2}$ Hard Tissue Pathology Unit, Matsumoto Dental University Graduate School of Oral Medicine, Shiojiri, Japan, \\ ${ }^{3}$ Department of Oral Surgery, Faculty of Dentistry, University of Health Sciences, Phnom Penh, Cambodia, \\ ${ }^{4}$ Formerly Unit of Stomatology, Cancer Research Centre, Institute for Medical Research, Jalan Pahang, Kuala Lumpur, Malaysia, \\ ${ }^{5}$ Department of Oral Pathology and Medicine, Graduate School of Medicine, Dentistry and Pharmaceutical Science, Okayama \\ University, Okayama, Japan
}

\begin{abstract}
The purpose of this report is to document a case of unsuspected ameloblastoma involving the right mandibular subpontic region in a 38-year-old Cambodian female patient. This lesion was purportedly preceded by multiple radiolucencies which were diagnosed as radicular cysts and treated a few times in the past years by enucleation followed by endodontic therapy of the affected teeth. Bridgework restoration of the partially edentulous area was performed. This case report demonstrates radiographic changes that occurred in the periods before and after the diagnosis of ameloblastoma. The case may represent an example of radicular cysts and ameloblastoma occurring as a collision phenomenon, or the ameloblastoma may have arisen as a result of neoplastic transformation of the lining epithelium in an inflammatory odontogenic epithelial cyst.
\end{abstract}

Key words: unsuspected ameloblastoma, cystic ameloblastoma, small ameloblastoma, radicular cyst, odontogenic cyst, neoplastic transformation

\section{INTRODUCTION}

The World Health Organization defined ameloblastomas as a slowly-growing, locally-invasive odontogenic epithelial neoplasm [1]. It is the second most common odontogenic tumor occurring without gender predilection and affecting a wide age range. The clinical, radiological and histological features of this neoplasm have been well-characterized [1, 2]. The general consensus is that the ameloblastoma originates in the enamel organ and its derivatives as well as in the epithelial lining of developmental odontogenic cysts $[1,3]$. Reports of ameloblastoma arising as a consequence of neoplastic transformation of the lining epithelium in an inflammatory odontogenic cyst are virtually unknown.

In this report, we present a case of ameloblatoma, which may represent an example of radicular cysts and ameloblastoma occurring as a collision phenomenon, or perhaps the ameloblastoma arose as a result of neoplastic transformation of the lining epithelium in an inflammatory odontogenic epithelial cyst.

\section{CASE REPORT}

History of present complaint: In January 2007, a 38year-old Cambodian female patient presented to the Department of Oral Surgery, Faculty of Dentistry, University of Health Sciences, Phnom Penh, Cambodia, with a complaint of painful swelling with pus discharge from her right lower jaw for the past one month.

Past dental history: The patient gave a history of removal of cysts from the same site a year earlier. According to the dentist attending to the patient, cystic lesions which looked clinically like radicular cysts were removed a few times. Based on the histopathology report of the specimens, the diagnosis was 'an inflammatory odontogenic cyst, most probably a periapical cyst (radicular cyst)'. However, these lesions kept recurring at the same site but seemed to have reduced in size following the last surgery. During the same period, the patient underwent endodontic treatment of her left mandibular central incisors, right mandibular central and lateral incisor, and right mandibular first premolar. Ceramic restorations of her right mandibular lateral incisor, right mandibular first premolar and right mandibular second molar as well, as bridgework replacing missing right mandibular canine and right mandibular second premolar and first molar, were also constructed. Her most recent history was of a painful swelling over the same area one month earlier, which improved after a course of antibiotics.

Clinical examination: Intraoral examination revealed a fluctuant swelling with buccal bony expansion over the right mandibular premolar-molar region and extending anteriorly along the labial alveolus. A discharging sinus was seen in the lingual mucosa between the right and left mandibular central incisors.

Radiographic examination: Orthopantomogram taken in 2006 showed a well-defined unilocular radiolucency located in the partially-edentulous body of the mandible, i.e. between the right mandibular first premolar and second molar (Fig. 1A). The radiolucency had mildly scalloped, sclerotic margins, and fine bony septa 

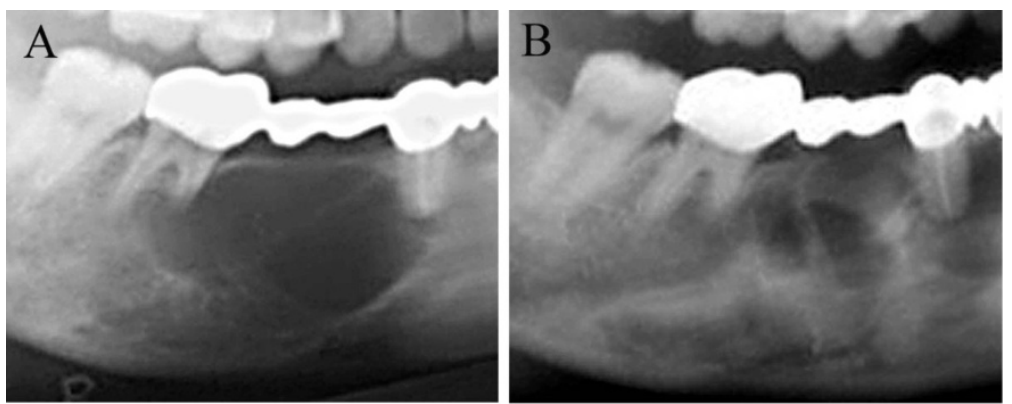

Fig. 1A. Radiograph (2006) showing a well-defined unilocular radiolucency located between the right mandibular first premolar and second molar.

Fig. 1B. Radiograph (January 2007) showing bone-like radiopacity in the original radiolucency between the right mandibular first premolar and second molar. Note a new unilocular radiolucency appears to have formed in the right mandibular canine region.
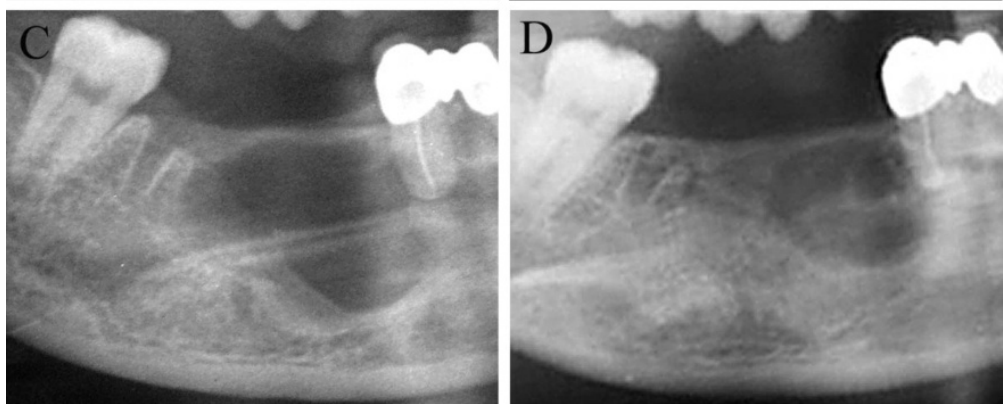

Fig. 1C. Radiograph (November 2007) showing bone-like radiopacity in the right mandibular canine region. A recurrent radiolucency has emerged between the right mandibular first premolar and second molar extraction socket and second molar.

Fig. 1D. Radiograph (June 2008) showing bone-like radiopacity in the right mandibular canine region. A recurrent radiolucency has emerged between the right mandibular first premolar and second molar extraction socket and second molar.

traversing the center. Root resorption of the right mandibular first premolar and second molar was evident.

The orthopantomogram taken in January 2007 showed considerable bone filling-in of the original radiolucency between the right mandibular first premolar and second molar (Fig. 1B). However, a new unilocular radiolucency appeared to have formed between the right mandibular lateral incisor and right mandibular first premolar. This new radiolucency extended anteriorly to the apices of the left and right mandibular central incisors. Radiographically it appeared to be separated from the original radiolucency by bony septa.

Biopsy: Under local anesthesia, the new radiolucent lesion was enucleated and submitted for histopathological examination. A diagnosis of 'cystic ameloblastoma' was made.

Follow-up radiographic examination: An orthopantomogram taken in November 2007 showed considerable bone-filling of the previously enucleated site, i.e. between the right mandibular lateral incisor and first premolar (Fig. 1C). However, a recurrent radiolucency had emerged between the right mandibular first premolar and second molar extraction socket. The superstructure spanning the right mandibular second premolar and first molar had been removed.

The orthopantomogram taken in June 2008 showed that the radiolucency between the right mandibular first premolar and second molar extraction socket had shrunk in size (Fig. 1D). The socket outline of the right mandibular second molar was still visible. There was no evidence of recurrence in the right mandibular canine region.

Treatment: In August 2008, surgical excision of the lesion from the mesial side of the right mandibular canine to the third molar was performed. The histopathology report stated that the lesion was a 'follicular ameloblastoma'. However, no further information regarding the outcome of this treatment was available as the patient was lost to follow-up.
Histopathological examination: Histopathologically, the soft tissue specimen enucleated from the right mandibular canine region in 2007 showed cystic features (Fig. 2A). The cystic wall mainly consisted of a dense fibrous connective tissue wall. In the cyst wall, there were some inflammatory cell infiltration especially in the inner layer. The lining epithelium, in parts, was some cell-layered cuboidal and/or squamous in shape (Fig. 2B). In most parts of the cyst wall, the lining epithelium showed odontogenic in shape, resulting ameloblastomatous proliferation. In particular, the most peripheral layer of cells was columnar with hyperchromatic nuclei, and lined up in a palisaded fashion. Furthermore, there were small ameloblastoma islands in the connective cyst wall, suggesting mural ameloblastoma (Fig. 2C, D). The intraluminar cells were sometimes loosely arranged. Furthermore, extensive proliferation occurred into the cystic spaces (Fig. $2 \mathrm{E})$. In the connective tissues which formed the cyst wall, there were budding features with occasional formation of ameloblastoma nests (Fig. 2F).

\section{Discussion}

Ameloblastoma is classified as a benign, locally-infiltrative odontogenic neoplasm, which is composed of proliferating odontogenic epithelial nests within a fibrous stromal tissue. Some variants have been subclassified as follows: solid/multicystic, extraosseous/ peripheral, desmoplastic, and unicystic [1]. Odontogenesis is a complex biological process, and this process is directly reflected in the development of odontogenic neoplasms, especially ameloblastomas. It is thought that the above-mentioned variants are due to the developmental complex system $[4,5]$.

Intraosseous small ameloblastomas that sometimes appear within the jawbone are not well-studied. The lesions are not clinically detected; therefore, clinicaloriented evidence is limited at present [6]. In the literature, there has been some discussion on the cellular sources of unsuspected small ameloblastoma arising in the jawbone. Furthermore, the oriented histogenesis is 

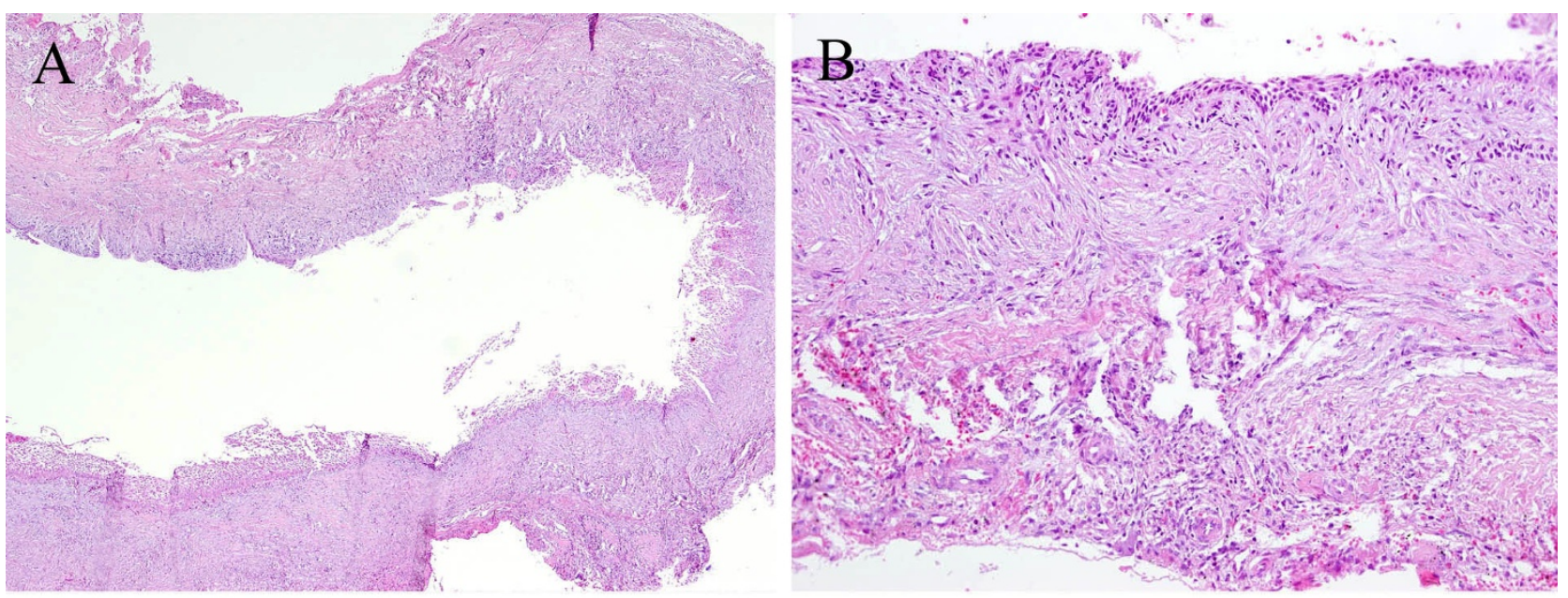

$\mathrm{C}$
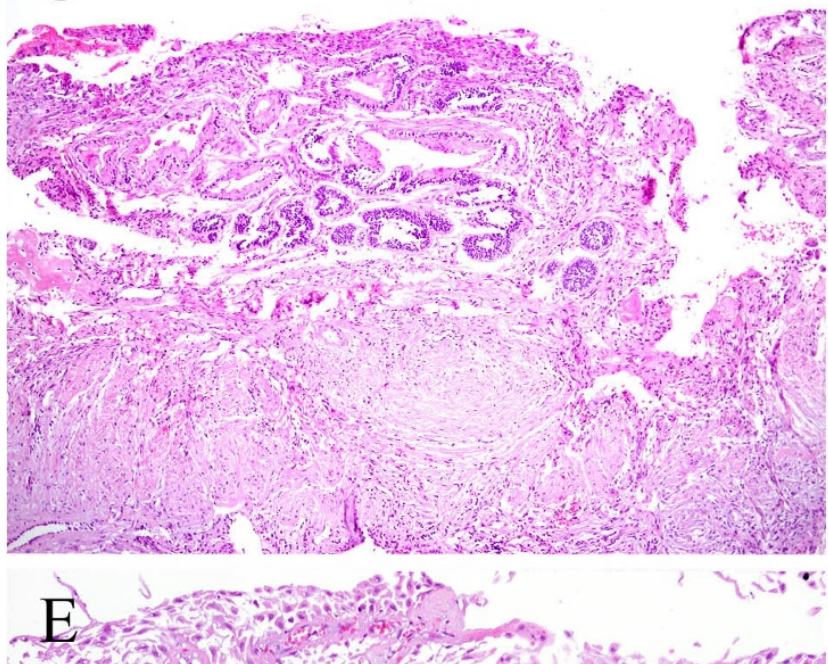
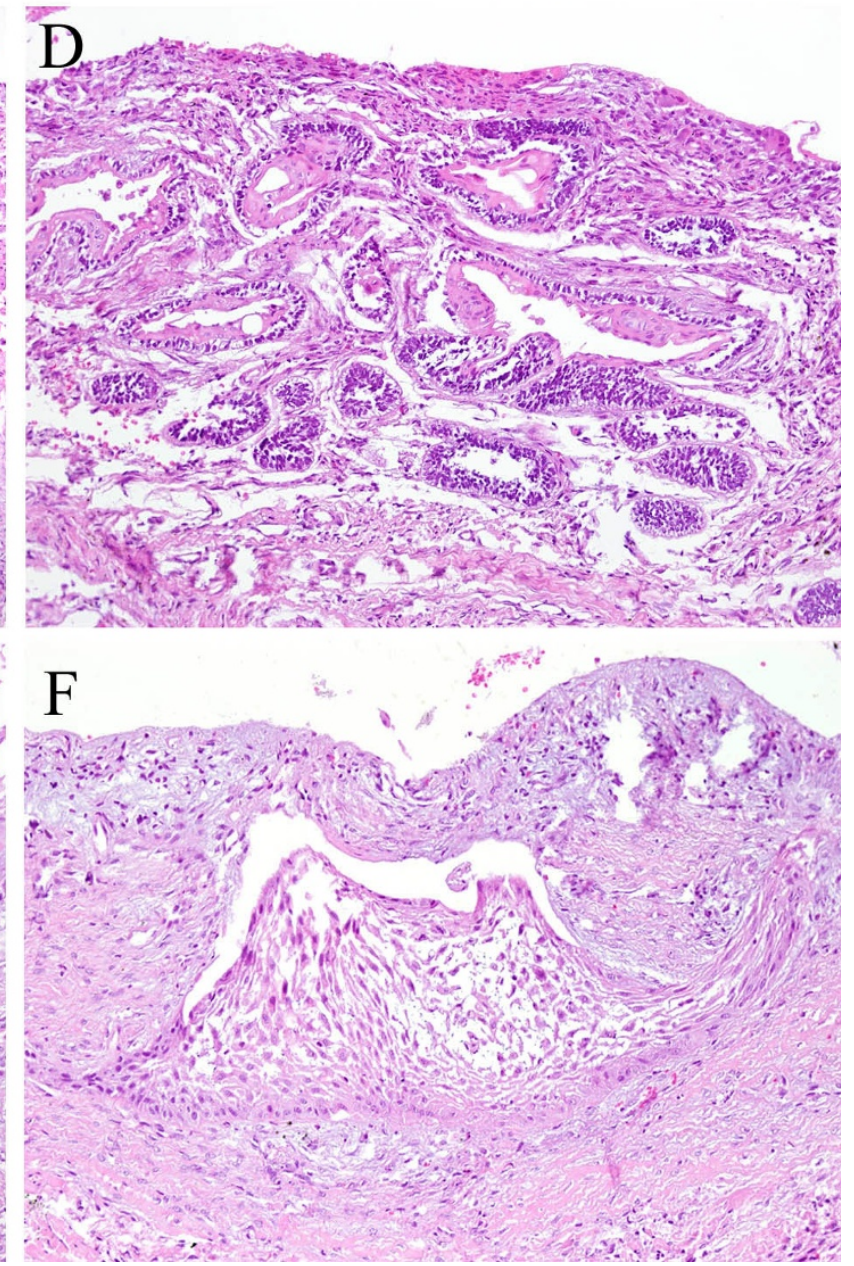

Fig. 2A. Low power view of the specimen showing cystic lesion with epithelial lining (original magnification x20).

Fig. 2B. Lining epithelium shows cuboidal and/or flattened in fashion (original magnification $\mathrm{x} 50$ ).

Fig. 2C. Small ameloblastoma islands in the connective tissue of the cyst wall (original magnification $x 50$ ).

Fig. 2D. Enlarged view of Fig. 2C showing follicular ameloblastoma nests (original magnification x100).

Fig. 2E. Ameloblastomatous proliferation of the lining epithelium and elongated and/or invaded proliferation of ameloblastoma in the connective tissue cyst wall (original magnification $\mathrm{x} 100$ ).

Fig. 2F. A follicular ameloblastoma island in the cyst wall (original magnification x100).

sometime discussed. Houston et al. (2007) [3] reported a case of ameloblastoma arising from dentigerous cyst. With regards to the neoplastic or atypical proliferative change from the lining epithelium of an odontogenic cyst, Antoh et al. (1993) [7] described such an example in a case of radicular cyst. Careful follow-up is necessary after treatment, since some cysts have the possibility of neoplastic transformation.

As mentioned previously, unsuspected ameloblastomas are usually small, asymptomatic and confined to 
the alveolar bone $[6,8]$. Their radiographic appearance is usually that of a nondescript lytic lesion. None of these features were observed in the current case. Instead, the case presented as an expansive fluctuant swelling associated with pain and a discharging sinus when the patient attended the Oral Surgery Clinic in January 2007. Radiographically, a unilocular lesion straddling the bone area between the right mandibular lateral incisor and first premolar was observed. Ameloblastoma remained unsuspected at this stage possibly because the clinical and radiological findings were nonspecific and could fit in a variety of inflammatory, neoplastic or cystic conditions of the jawbone.

With regards to the current case, the precise relationship between the radicular cysts enucleated from the right mandibular premolar-molar region in 2006, ameloblastoma diagnosed in the right mandibular canine region in January 2007, and follicular ameloblastoma in the right premolar-molar region in August 2008 remains an enigma. It is likely that the ameloblastoma located in the right mandibular canine and premolar-molar regions are one and the same lesion. Although plain radiographs showed a bony septa separating the two lesions, cancellous spread not detectable on plain radiographs may have occurred. The link, if any, with the radicular cysts that preceded the diagnosis of ameloblastoma in the right mandibular premolar-molar region is also unclear. Evaluation of the past dental history of this case showed that the first lesion diagnosed histopathologically was an apical inflammatory odontogenic cyst (radicular cyst) and this was removed a few times. In January 2007, the lesion enucleated from the right mandibular canine region was histopathologically examined and diagnosed as a cystic ameloblastoma. In consideration of the close proximity of the ameloblastoma in the site where the radicular cysts were previously enucleated. We therefore speculated that this case possibly represented an example of ameloblastoma arising from a cyst. We theorized that the cyst lining epithelium progressed and underwent ameloblastomatous change. Histopathologically this is a feasible concept because the epithelial cell rests of Malassez, which gives rise to radicular cyst, have been implicated in the origin of multiple odontogenic cysts and neoplasms [12]. In addition, the histopathological examination results are consistent with the above consideration. Furthermore, we observed small follicular ameloblastoma islands in the cyst wall connective tissues. The final histopathological diagnosis was follicular ameloblastoma. Another plausible explanation is that these entities may represent a collision phenomenon. The occurrence of collision lesions, including those of an odontogenic epithelial nature, is not uncommon in the jawbones [9-13]. That only radicular cysts were enucleated and diagnosed in 2006 may be due to the fact the ameloblastoma developing in the same area was in its incipient stage and therefore undetected.

In summary, this case reported here may represent an example of radicular cyst and ameloblastoma occurring as a collision phenomenon, or of an ameloblastoma arising from a result of neoplastic transformation of the lining epithelium in an inflammatory odontogenic (radicular) cyst. Although, their true rela- tionships remain unknown, nonetheless the considered pathogenesis was consistent with the course of histopathology and radiography.

Acknowledgments: This research was supported in part by a Grant-in Aid for Scientific Research (C) (20592349) from the Japan Society for the Promotion of Science.

\section{REFERENCES}

1. Gardner DG, Heikinheimo K, Shear M, Philipsen HP, Coleman H. Ameloblastomas. In Barnes L, Eveson JW, Reichart P, Sidransky D ed. World Health Organization Classification of Tumours. 296-300, 2005, IARC Press, Lyon, France.

2. Reichart PA, Philipsen HP, Sonner S. Biological profile of 3677 cases. Eur J Cancer B Oral Oncol. 1995; 31B(2): 8699.

3. Houston GD. Oral Pathology: Ameloblastoma arising from dentigerous cyst. J Okla Dent Assoc. 2007; 98(9): 28-29.

4. Kawakami T, Nagatsuka H. Cell differentiation of neoplastic cells originating in the oral and craniofacial regions. pp1-56, 2009, Nova Science Publishers, NY, USA.

5. Nakano K, Nagatsuka H, Tsujigiwa H, Gunduz M, Katase N, Siar CH, Kawakami T. Immunohistochemical characteristics of odontogenic neoplasms and their physiological counterparts. J Hard Tissue Biol. 2008; 17: 79-90.

6. Ide F, Mishima K, Yamada H, Horie N, Saito I, Shimoyama T, Kusama K. Unsuspected small ameloblastoma of the alveolar bone: A collaborative study of 14 cases with discussion of their cellular sources. J Oral Pathol Med. 2008; 37(4): 221-227.

7. Antoh M, Hasegawa H, Kawakami T, Kage T, Chino T, Eda S. Hyperkeratosis and atypical proliferation appearing in the lining epithelium of a radicular cyst: report of a case. J Cranio-Maxillofac Surg. 1993; 21: 210-213.

8. Tarsitano JJ, Holmes PJ. Unsuspected ameloblastoma: report of case. J Am Dent Assoc. 1973; 87(4): 898-900.

9. Han PP, Nagatsuka H, Siar CH, Tsujigiwa H, Gunduz M, Tamamura R, Borkosky SS, Katase N, Nagai N. A pigmented calcifying cystic odontogenic tumor associated with compound odontoma: a case report and review of literature. Head Face Med. 2007; 3: 35 -41.

10. Siar $\mathrm{CH}, \mathrm{Ng} \mathrm{KH}$, Chia TY. Combined granular cell ameloblastoma and plexiform granular cell odontogenic tumour. Sing Dent J. 1990; 15: 35-37.

11. Siar $\mathrm{CH}, \mathrm{Ng} \mathrm{KH}$. Combined ameloblastoma and odontogenic keratocyst or keratinizing ameloblastoma. Br J Oral Maxillofac Surg. 1993; 31: 183-186.

12. Bascones J, Llanes F. Clear cells in epithelial cell rests of Malassez. Oral Oncol. 2005; 41: 99-100.

13. Siar $\mathrm{CH}$, Oo VPA, Nagatsuka H, Nakano K, Ng KH and Kawakami T. Angiogenic squamous dysplasia-like phenomenon in oral epithelial precursor lesions. Eur J Med Res. 2009; 14: 315-319.

Received: June 25, 2009 / Accepted: July 24, 2009

Address for correspondence:

Keisuke Nakano, DDS, PhD,

Hard Tissue Pathology Unit,

Matsumoto Dental University

Graduate School of Oral Medicine,

1780 Hirooka-Gobara,

Shiojiri, 399-0781 Japan

Phone and Fax: +81-(0)263-51-2035

E-mail: $\quad$ keisuke1@po.mdu.ac.jp 